\title{
El sismoscopio eléctrico
}

J Rafael Urdaneta B

Rev. Acad. Colomb. Cienc. Ex. Fis. Nat. 1954, 9 (35): 227-232.

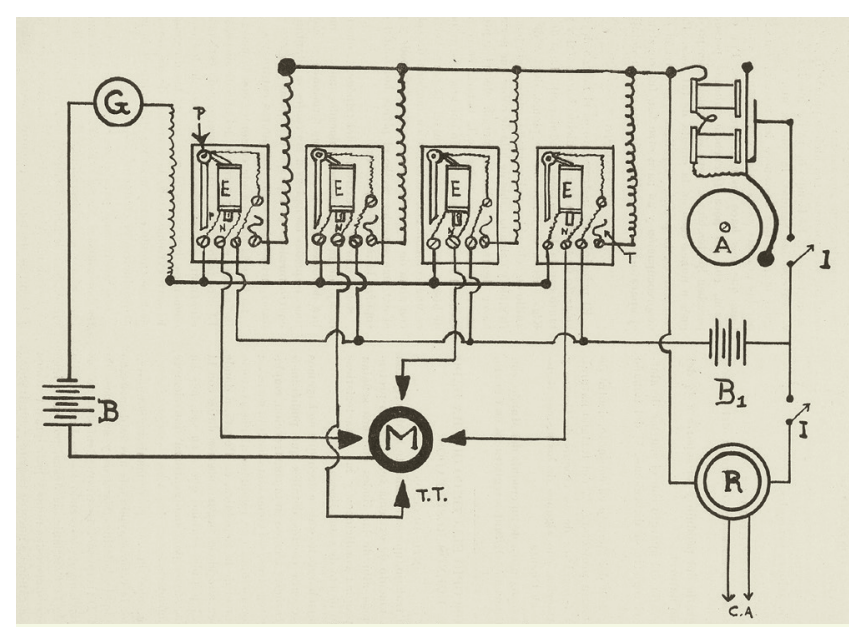

Diagrama del detector sísmico.

Indicaciones: G. Galvanómetro; P. Palancas; N. Núcleos móviles; E. Electroimanes; T. Trinquetes; T.T. Tornillos de tope; A. Timbre de alarma; R. Reloj; M. Masa del péndulo; B. - B1. Baterias; I. Interruptores

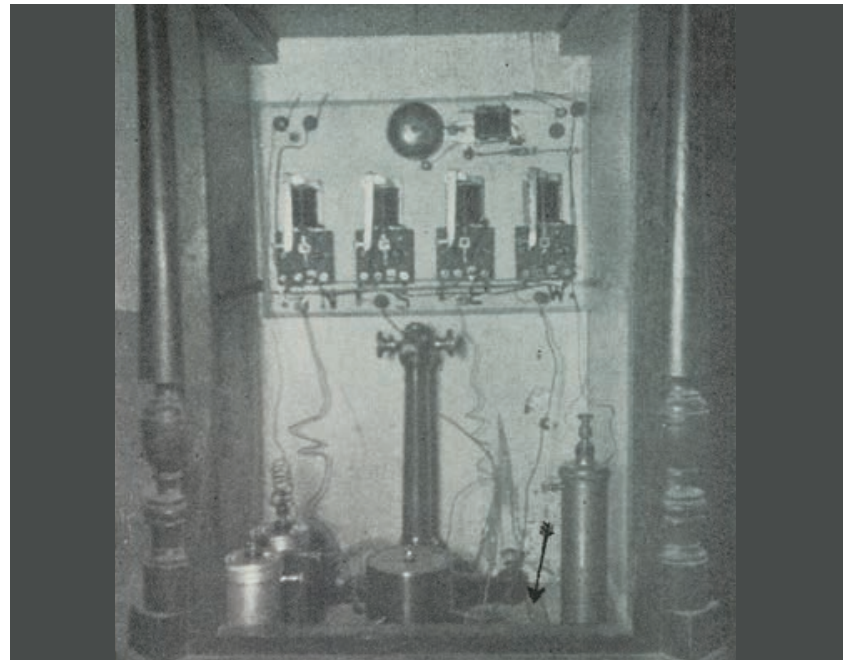

Fotografía del sismoscopio eléctrico dentro de la caja protectora; se observan con claridad casi todos sus detalles (Véase lamina 4a dentro del artículo clásico "El sismoscopio eléctrico"
Este articulo escrito por Rafael J Urdaneta en el año 1953 presenta el detalle de un detector sísmico construido en la ciudad de Cúcuta, Norte de Santander.

El principio del instrumento es el mismo del sismógrafo de péndulo de movimiento horizontal con una masa pendular cilíndrica de 3,5 kilogramos en plomo fundido, dentro de un anillo de cobre bien pulimentado, suspendido por un hilo de cobre flexible y delgado que se conecta con energía electrica proveniente de una batería. El soporte es una base trípode de hierro con sus tornillos de nivel. La parte vertical es una columna cónica de cobre con alma de acero que se atornilla al trípode y por el extremo superior recibe el pivote horizontal que soporta la masa del péndulo. Las componentes de los puntos cardinales N, S, E, W se registran mediante un electroimán vertical de núcleo móvil en cada uno de los mecanismos. Los núcleos móviles de los electroimanes al ser atraídos, golpean fuertemente las armaduras con el consiguiente ruido $\mathrm{y}$, además, el galvanómetro marca cada contacto.

Mediante graficas hechas a mano y fotografías, Urdaneta presenta el desarrollo en detalle del Sismoscopio eléctrico construido y en un mapa de Suramérica algunos de los sismos registrados en Cúcuta, mediante el equipo diseñado y construido. En los sismos pequeños ocurridos en Cúcuta, el Sismoscopio indico dirección y sentido bien definido. El registro total desde abril de 1952 hasta julio de 1953 fue de 103 temblores.

Para el objetivo que se propuso con la construcción del aparato, Urdaneta considera que la principal era que registrase temblores mas o menos intensos y cercanos. Mediante la comunicación con el Instituto Geofísico de los Andes, en Bogotá, Urdaneta considera que cambio la manera de apreciar las cosas y les obligo a una mas cuidadosa observación que se tradujo en constantes reformas, mejoras en las condiciones de emplazamiento, seguridad en los registros y control de la hora, mediante las señales transmitidas por la Radiodifusora Nacional, amen de una permanente revisión de las baterías eléctricas. En resumen, este fue un trabajo de sinergia entre diferentes instituciones para avanzar en la ciencia y la tecnología de la sismografia en Colombia, manteniendo el aparato en perfecto estado de funcionamiento.

Horacio Torres Sánchez, Profesor Emérito UN Miembro de Número 


\section{EL SISMOSCOPIO ELECTRICO}

POR J. RAFAEL URDANETA B.

Detalle de un detector sísmico construido en Cúcuta, Norte de Santander-Colombia, por el autor de estas notas.

1-Introducción.

CONTENIDO:

2-El aparato.

3-Emplazamiento.

4-Consideraciones generales.

5-Conclusión.

ILUSTRACIONES :

1-Copia heliográfica del diagrama del sismoscopio.

2-Fotografía del péndulo y su soporte a $1 / 4$ del tamaño natural.

3--Fotografía del aparato con el sistema de alarma y el registro de direcciones. $1 / 4$ del tamaño natural.

4-Fotografía del aparato en conjunto, dentro de la cubierta protectora. $1 / 4$ del tamaño natural.

5-Copia fotográfica de un croquis de Centro y Sur-América, con algunos de 10 sismos registrados en Cúcuta.

6-Copia fotográfica de un croquis del Mundo, con los sismos más distantes registrados en Cúcuta.

\section{UN SISMOSCOPIO ELECTRICO QUE HA DADO BUENOS RESULTADOS}

\section{INTRODUCCION}

Con este trabajo, que es solamente la descripción de un instrumento de extrema sencillez, no pretendemos ir más allá de las lindes que nos señalan nuestras propias condiciones y las características del aparato objeto de estas líneas.

Esto, sin embargo, no quiere decir que no hayamos tenido en cuenta ciertos detalles que pudiéramos llamar de carácter técnico en la construcción, emplazamiento y registros, así como una absoluta seriedad en las informaciones que rendimos al observatorio sísmico central de la República, en Bogotá, a donde hemos procurado hacer llegar nuestros datos con la oportunidad posible, entidad que a su vez, nos ha enviado los suyos por una gentileza que siempre sabremos agradecer.

Sea ésta, oportunidad para manifestar nuestra gratitud por la valiosa ayuda que nos ha dispensado con el envío de boletines sísmicos y sus voces de aliento, el Director del Instituto Geofísico de los Andes Colombianos, Reverendo Padre Jesús Emilio Ramírez, de la Compañía de Jesús, por cuyo insinuación escribimos estas notas y para quien tenemos la expresión cálida de nuestro respeto y admiración.
Después del terremoto de Arboledas, Cucutilla y Salazar, en el Norte de Santander, toda la región continuó por largo tiempo en un estado de agitación sísmica relativamente considerable, lo cual nos decidió a construír un mecanismo que, sin ser un sismógrafo, al funcionar nos hiciese pensar ipso facto, en un temblor. El tiempo borra las huellas de dolor y cicatriza las heridas, lo que es una ventaja, de igual manera que desvanece los temores causados por las grandes catástrofes, circunstancias esta última que nos hace vivir desprevenidos. Por esta razón, con no poca frecuencia muchas personas perciben los temblores, pero sólo mucho tiempo después o nunca caen en la cuenta de tal cosa. Para eliminar en lo posible el peligro que entraña esta despreocupación y no para otro fin, fue construído el aparato que a continuación pasamos a detallar.

\section{EL APARATO}

El principio del instrumento es el mismo del sismógrafo de péndulo de movimiento horizontal y por tanto consta de una masa pendular cilíndrica de $\mathbf{3 . 5}$ Klgs., hecha de fundición de plomo, dentro de un anillo de cobre bien pulimentado, suspendida por un flexible y delgado cable de hilo de cobre que permite hacer llegar hasta ella la energía eléctrica proveniente de uno de los polos de la batería correspondiente. El soporte está formado por una base trípode de hierro con sus tornillos de nivel; la parte vertical es una columna un tanto cónica también de cobre, con alma de acero que se atornilla al trípode y por el extremo superior recibe el pivote horizontal que soporta la masa del péndulo.

Sobre uno de los extremos del trípode, el que está debajo de la masa, hemos colocado una cruz metálica de brazos iguales en cuyas terminales se hallan unas pequeñas escuadras a las cuales se adaptan los cuatro tornillos de tope que circundan la masa pendular, orientados según los puntos cardinales.

La batería mencionada comunica con la masa por la base del soporte y a través del hilo de suspensión. El otro polo va a un galvanómetro $\mathrm{y}$ de éste pasa a alimentar un extremo de cada uno de los cuatro elementos destinados al registro de las direcciones. El otro extremo recibe la corriente de los tornillos de tope cuando éstos, a su vez, la toman de la masa al producirse el contacto.

Los mecanismos para el registro de las componentes horizontales N. S. y E. W., están constituídos cada uno, por un electroimán vertical de núcleo móvil, el cual se halla siempre hacia la parte inferior de la bobina. Al producirse el contacto entre masa y tornillos de tope, el circuito queda cerrado y el núcleo atraído violentamente, va a dar contra el 


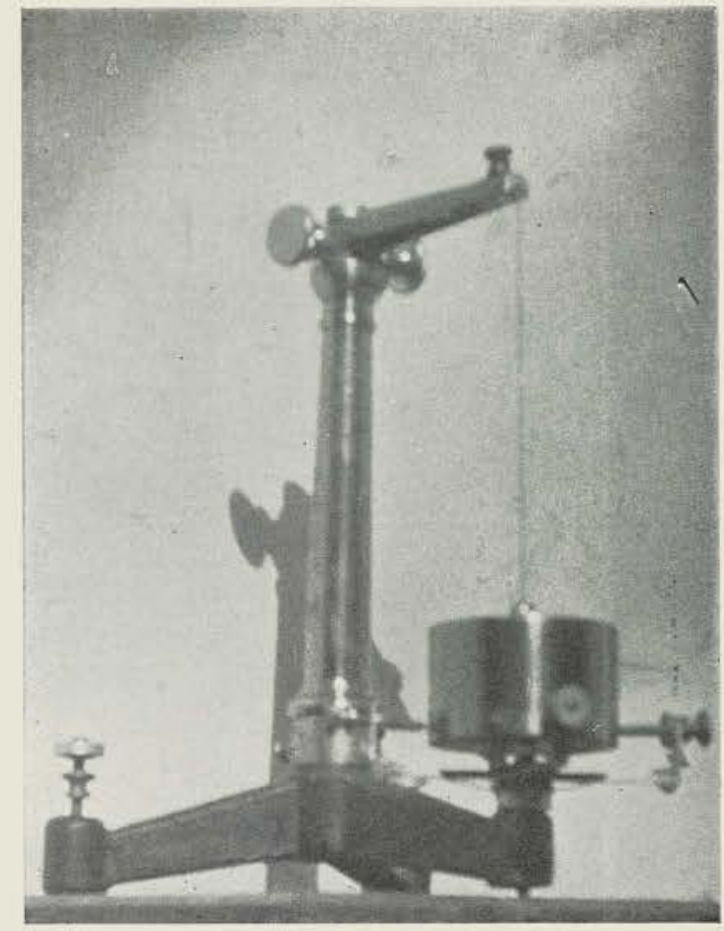

L.AMINA $2^{\mathrm{a}}$

Detalle del péndulo con su soporte general, en el cual pnede observarse con suficiente claridad su estructura, las distintas jartes que forman tales como los tormillos de tope el hilo de suspensión. la colocación de la masa, efc.

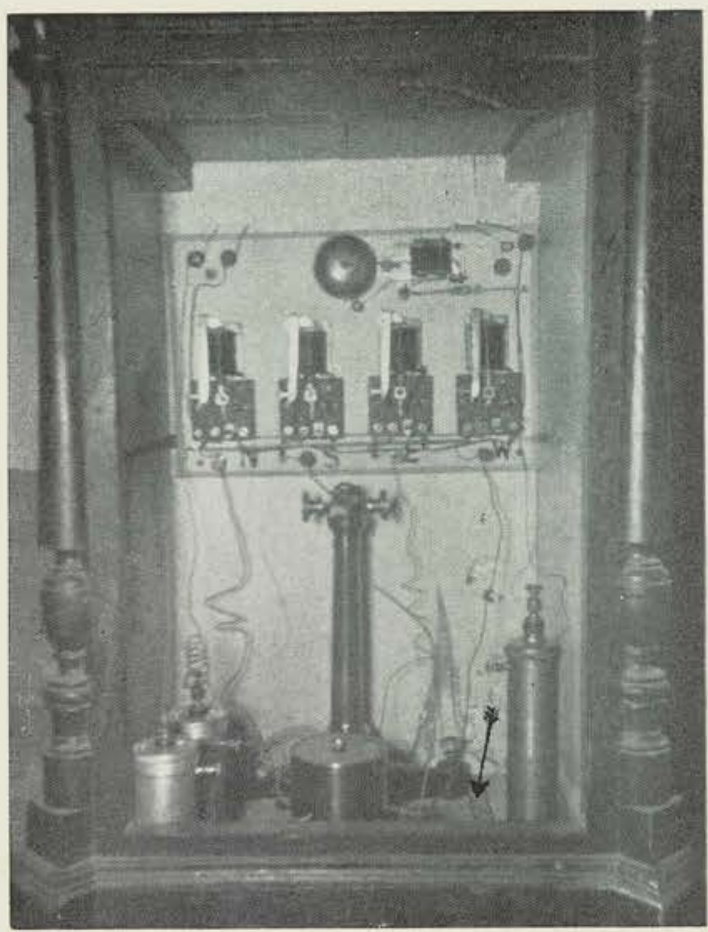

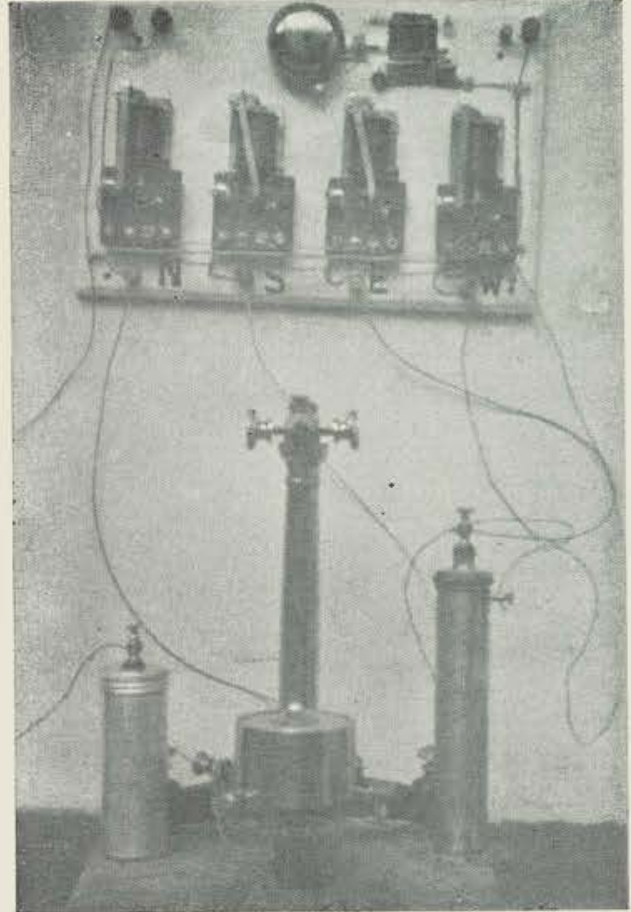

LAMINA $3^{\mathrm{a}}$

Fotografia del aparato en conjunto, en la cual se muestran : el péndulo, las baterias eléctricas y los sistemas de alarma y registro de las direcciones. Alli puede apreciarse como son retenidas las palancas por los trinquetes al producirse ef rontacto en los topes respectivos.
L.A.MIN.A $4^{\mathrm{a}}$

Fotografía del sismoscopio eléetrico dentro de la caja pro tectora: se observan con claridad casi todos sus detalles: los conductores de la izquierda que salell por la parte superior van al galvanómetro: los de la derecha al cortacircuitos, La flecha muestra la separación entre el bloque de cemento en que reposa el péndulo, separación que aparece rellena con tubo de papel parafinado. La batería de la i\% quierda corresponde al sistema de registro de direcciones y la de la derecha al cireuito de al:arma. 


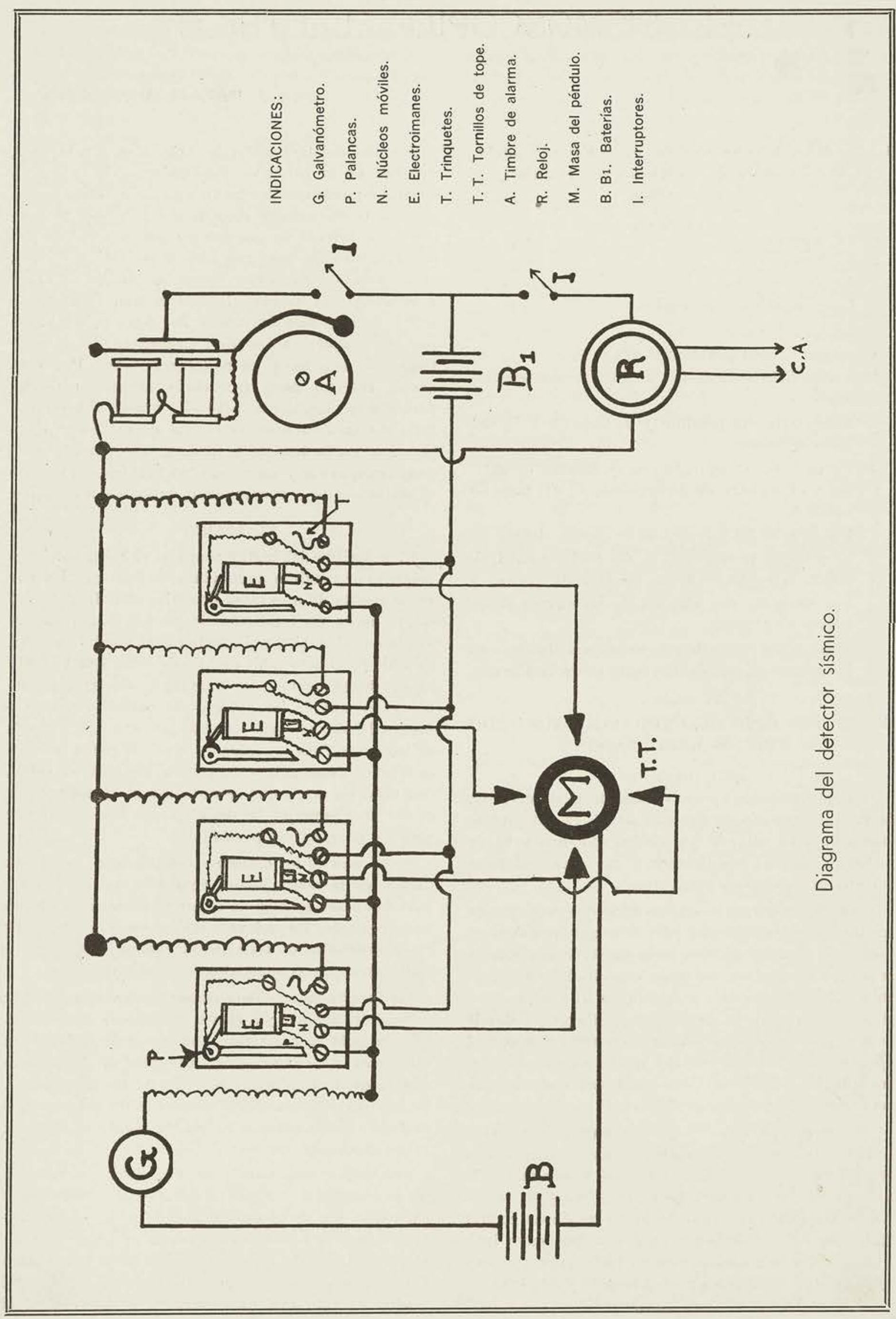




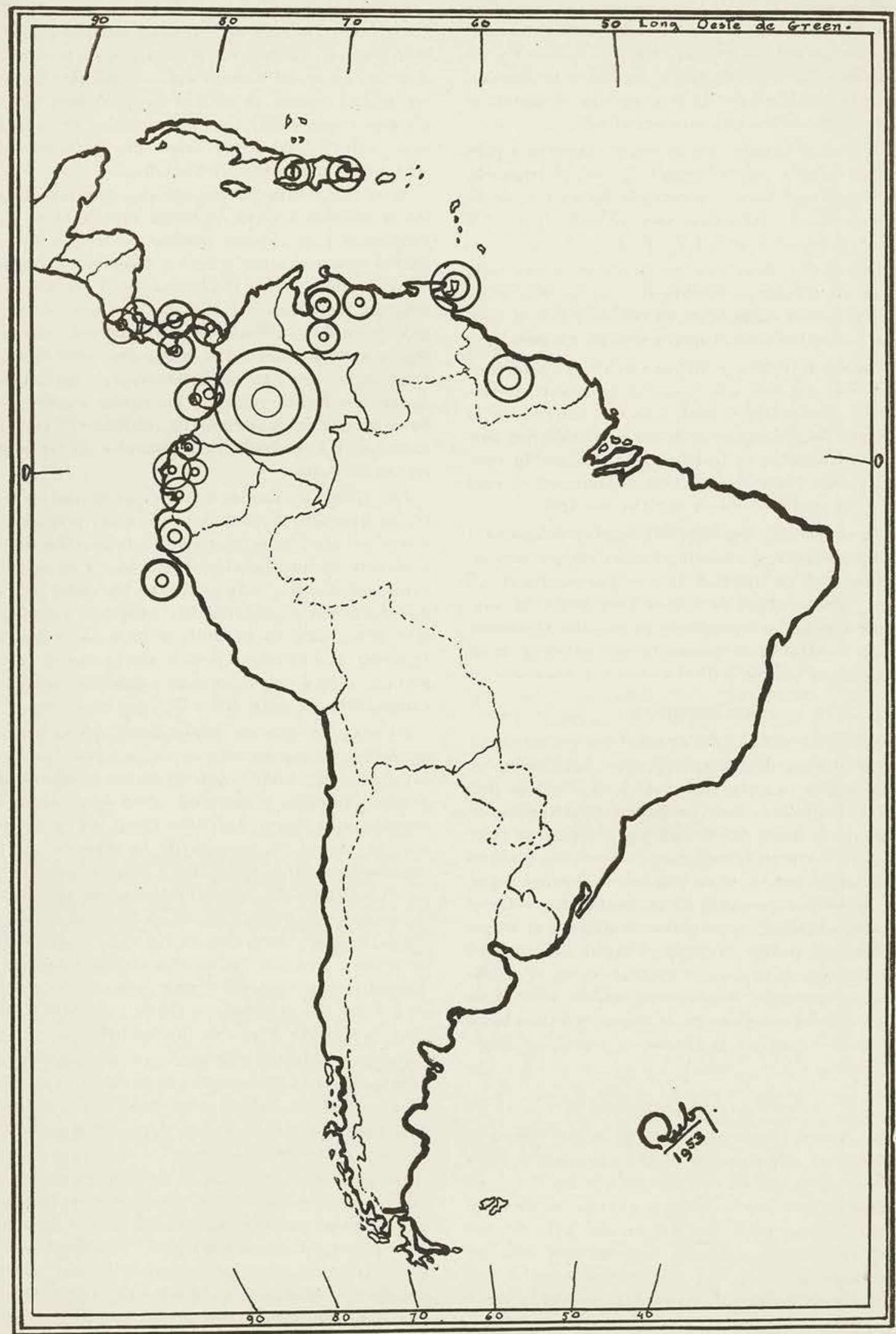

LAMINA $5^{\mathrm{a}}$

Algunos de los sismos Centro y Sur-Amerícanos registrados en Cúcuta, mediante el sismoscopio eléctrico. Las distancias epicentrales fueron calculadas por el Instituto Geofísico de los Andes Colombianos, en Bogotá. La localización en el croquis es aproximada. 


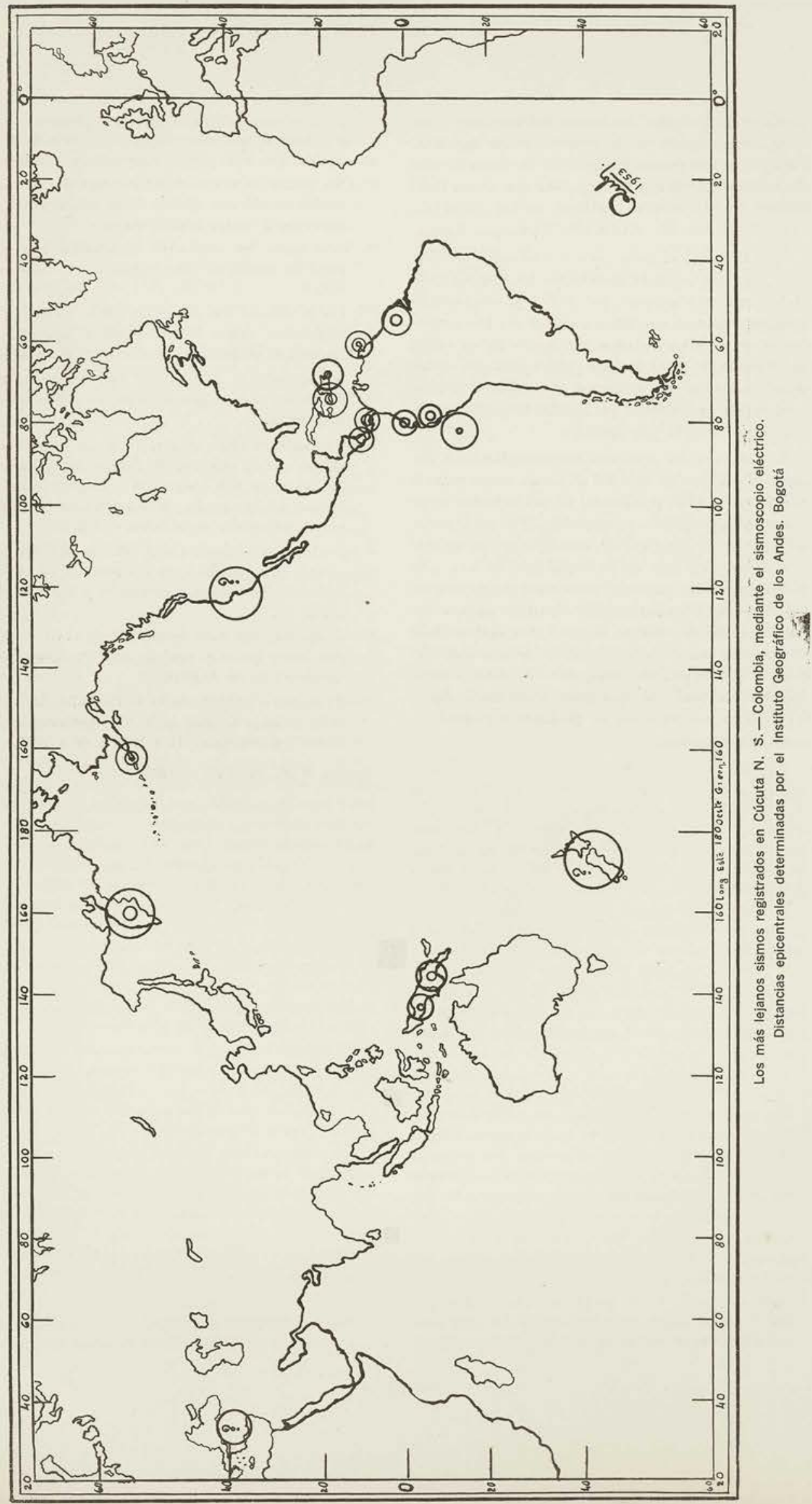


brazo de una palanca que reposa precisamente sobre el extremo opuesto de la bobina. En estas condiciones la palanca es lanzada hacia la derecha y un trinquete adecuado impide su regreso a la posición primitiva, marcándose de esta manera el sentido o la dirección del movimiento registrado.

Existe otra batería uno de cuyos extremos o polo va directo a la palanca citada $\mathrm{y}$ por el trinquete de retención al borne de entrada de un timbre de alarma. El otro polo pasa por un corta-circuito y de éste al borne de salida del timbre.

Como puede observarse, la palanca en cuestión, además de señalar la posible dirección o sentido de un movimiento, al ponerse en contacto con el trinquete hace funcionar el mecanismo de alarma.

Consiste el tercero y último circuito en una derivación del anterior que, acciona un nuevo electroimán de núcleo fijo el cual, a su vez, interrumpe la corriente de alimentación de un reloj eléctrico destinado al registro de la hora. Una ilustración completa puede darla el diagrama adjunto, en el cual está comprendido todo el sistema descrito.

Hemos preferido dos circuitos independientes a fin de obtener un funcionamiento continuo del correspondiente al de alarma, ya que por medio de la masa y sus tornillos de contacto sería inteimitente y podría pasar desapercibido en muchas ocasiones, además de debilitarse mucho la corriente por la resistencia que ofrecía todo el sistema mencionado.

\section{EMPLAZAMIENTO}

El reducido tamaño del aparato nos permite mantenerlo dentro de nuestra propia habitación, en donde se hizo una excavación de unos $50 \mathrm{~cm}$. de profundidad por 40 de lado; se hundieron 4 varillas de hierro de $3 / 8$ hasta $\operatorname{los} 80 \mathrm{~cm}$. y por último se construyó un bloque de concreto. Para evitar posibles movimientos por causa de pisadas, baldosines flojos, etc., dejamos un espacio de un centímetro entre el piso de la habitación propiamente dicho y el bloque destinado a recibir la parte eesncial del aparato. Una cubierta de madera lo protege contra el viento, polvo, animales y demás que puedan alterar su normal desempeño y dentro de la cual se hallan todos los componentes del instrumento, excepción hecha del reloj.

\section{CONSIDERACIONES GENERALES}

De acuerdo con el propósito para que fue construído, nos decidimos por el período corto y masa pequeña. Y si bien consideramos que los desplazamientos del péndulo se reducen cuando los períodos propio y de perturbación son iguales pero de fase opuesta, llegamos a la conclusión de que esta circunstancia no tendría mayor influencia en el caso de temblores de alguna intensidad y de origen cercano.

Por otra parte, como no posee sistema alguno de amplificación, los contactos se hallan a muy reducida distancia de la masa, $1.0 \mathrm{~mm}$. como máximo.
En vía de ensayo adaptamos los registros N. S. y E. W., pero después de repetidas observaciones vimos que era posible, en ciertas circunstancias, la determinación del sentido del movimiento. Ya con las modificaciones necesarias comprobamos que en algunos casos, efectivamente resultaba afectado un solo contacto, es decir, había discriminación del sentido del movimiento registrado.

Asimismo hemos podido apreciar que los contactos se suceden a veces en forma rápida, otras pausadamente y en algunos casos se prolonga sobre un mismo tope por espacio de 3 y 4 segundos. Los núcleos movibles de los electroimanes al ser atraídos, golpean fuertemente las armaduras con el consiguiente ruido $\mathrm{y}$, además, la aguja del galvanómetro marca cada contacto. De suerte que estas dos circunstancias nos han permitido captar los detalles a que nos hemos referido, los cuales sugieren un buen número de conjeturas en relación con la naturaleza de los movimientos registrados en las condiciones anotadas.

Por último, en los dos o tres casos en que los temblores han sido percibidos por muchas personas, el cierre del circuito se ha hecho en todas direcciones, a manera de un verdadero bamboleo; y en aquellos otros notados por muy pocos, se ha hecho por los dos contactos diametralmente opuestos. En el primer caso, como es natural, se hace imposible determinar por nuestros medios mecánicos dirección alguna; y en el segundo, cabe solamente indicar la componente afectada, NS o EW, según el caso.

Para el fin que nos propusimos al construir el aparato, sólo una de todas estas consideraciones nos interesaba en realidad: que registrase temblores más o menos intensos y cercanos. Pero establecidas comunicaciones con el Instituto Geofísico de los Andes, en Bogotá, el aspecto de la cuestión cambió nuestra manera de apreciar las cosas y nos obligó a una más cuidadosa observación que se traduce en constantes reformas, mejoras en las condiciones de emplazamiento, seguridad en los registros y control de la hora mediante las señales transmitidas por la Radiodifusora Nacional y una permanente revisión de las baterías eléctricas, a fin de mantener el aparato en perfecto estado de funcionamiento.

La gran sencillez y lo elemental del instrumento saltan a la vista, así como la tosquedad de su estructura general, bien clara en las fotografías adjuntas. Con todo, los resultados no dejan de ser satisfactorios y hasta interesantes.

De un mecanismo como el descrito no es posible esperar ni exigir más de lo que ha dado. El número de temblores registrados, sus levísimas intensidades y las grandes distancias del origen de varios de ellos, son suficientes para hacernos sentir relativamente satisfechos. Al menos la finalidad primordial ha sido cumplida con pleno éxito.

\section{LAS GRAFICAS}

Nos referimos exclusivamente a los mapas con los cuales hemos pretendido ilustrar estas anotaciones, 
porque consideramos conveniente destacar que, ellos se refieren propiamente al número de los registros, a los países y al distanciamiento de las zonas de conmoción con relación a Cúcuta, más que a nna localización exacta en tales gráficas, de los epicentros que en todo caso han sido determinados por Bogotá.

Para llevarlos al mapa hemos utilizado los boletines sísmicos que gentilmente nos ha suministrado el Insituto Geofísico de los Andes y comparando nuestros registros con ellos, sacamos los que coinciden en fecha $\mathbf{y}$ hora; luégo por medio de un esfera de 18 pulgadas localizamos aquellos para los cuales Bogotá ha dado una situación geográfica completa, y en forma aproximada los trasladamos al croquis general del mundo y de América.

No poseemos los boletines correspondientes a noviembre y diciembre de 1952 ni los de enero y parte de febrero de 1953 , por lo cual 17 movimientos regis. trados no han podido computarse. Por otra parte, muchos de los que pueden considerarse con epicen. tros dentro del área de la República, no han sido detallados en los mapas y nos hemos limitado a señalarlos con 3 circunferencias céntricas para no recargar el dibujo. Además, parece haber equivocación por nuestra parte, en la anotación de una o dos fechas, lo que no pudimos comprobar de manera satisfactoria; de aquí que aparezcan en el mapa, dos o tres sismos con carácter de probable o posible registro por nosotros.

\section{CONCLUSION}

Para concluir, queremos dejar constancia de algunos puntos que nos parece conveniente consignar:

19-No pasan de 6 los temblores notados por las gentes en Cúcuta. Entre éstos, solamente 3 alcanzaron a causar alguna alarma.

$2^{9}$ - Casi todos los temblores registrados han sido para la localidad muy pequeños e imperceptibles.

$3^{\circ}$-Los temblores con origen en Chile, Bolivia y la Argentina, nunca han afectado el aparato, no importa el carácter destructor que hayan tenido.

$4^{\circ}$ - No obstante lo anterior, se han registrado movimientos cuyo origen se hallaba a considerables distancias.

$5^{\circ}$ - No han coincidido los temblores, en forma indudable, con el registro de fuertes temperaturas locales ni se han observado alteraciones en las curvas del barógrafo, en coincidencia con movimientos sísmicos registrados en Cúcuta.

$6^{\text {O }}$ - En los sismos pequeños y aun en aquellos percibidos por personas en los pisos altos, el sismoscopio ha indicado dirección y sentido bien definidos.

$7^{9}$-El aparato funciona desde el 20 de abril de 1952, con interrupciones prolongadas en agosto, septiembre y parte de octubre.

$8^{\circ}$-El registro total desde la fecha indicada, hasta el 21 de julio de 1953 es de 107 temblores, de los cuales corresponden 47 a 1952 y 60 a 1953 .

Cúcuta, N .S., julio 25 de 1953. 